\begin{tabular}{|l|l|l|l|l|l|l|}
\hline Rev. Intropica & ISSN 1794-161X & Vol. 12 & No. 2 & 117 - 130 & julio - diciembre de 2017 & Santa Marta, Colombia \\
\hline
\end{tabular}

\title{
EFECTOS DE LA ENTRADA DE AGUA DEL RÍO MAGDALENA EN LA PRODUCCIÓN PRIMARIA DEL FITOPLANCTON EN LA CIÉNAGA PAJARAL, CARIBE COLOMBIANO
}

\author{
EFFECTS OF THE WATER INLET FROM MAGDALENA RIVER ON THE \\ PHYTOPLANKTONIC PRIMARY PRODUCTION IN THE CIÉNAGA PAJARAL, COLOMBIAN \\ CARIBBEAN
}

Carlos Augusto Hernández-Jiménez

\begin{abstract}
RESUMEN
Se evaluó la producción primaria del fitoplancton en la Ciénaga Pajaral, entre agosto de 1999 y octubre de 2000, con el propósito de monitorear los efectos de la entrada de agua dulce a través del caño Aguas Negras. Se utilizó el método de botellas claras y oscuras para valorar la producción primaria; también se estimaron las siguientes variables: concentración de clorofila $a$ y feofitina $a$, conductividód eléctrica del agua, ortofosfatos, nitrógeno inorgánico total y $\mathrm{CO}_{2}$. Los valores más altos ocurrieron en la Ciénaga de Pajaral (máxima producción primaria neta $=10,19 \mathrm{gC} \cdot \mathrm{m}^{-2} \cdot$ día ${ }^{-1}$ ) y los menores en el caño Aguas Negras. La respiración de la comunidad planctónica fue en algunos casos mayor que la producción primaria neta. Se observó una relación significativa entre la salinidad del agua y los valores de producción primaria neta y de concentración de clorofila $a$. Además, se estableció la composición de especies y la abundancia de fitoplancton.
\end{abstract}

PALABRAS CLAVE: producción primaria; fitoplancton; lagunas costeras

\begin{abstract}
Phytoplanktonic primary production in the Ciénaga Pajaral was evaluated between August 1999 and October 2000, to monitor the effects of freshwater inlet from the Aguas Negras Channel. The light and dark bottles method was used to assess the primary production. Chlorophyll $a$, pheophytin $a$, electrical conductivity, salinity, orthophosphate, total inorganic nitrogen, and carbon dioxide were also measured. Higher values of net primary production (NPP) are reported in the Ciénaga Pajaral (maximum value of $\left.10.19 \mathrm{gC} \cdot \mathrm{m}^{-2} \cdot \mathrm{dia}^{-1}\right)$ in comparison with the ones reported in Aguas Negras Channel. The plankton community respiration was sometimes greater than the net primary production. Significant correlations between salinity and NPP and between salinity and chlorophyll $a$ were found. Furthermore, species composition and phytoplankton abundance were determined.
\end{abstract}

KEY WORDS: primary productivity; phytoplankton; coastal lagoons 


\section{INTRODUCCIÓN}

La entrada de energía a la biosfera y a cualquier sistema biológico está dada por el proceso fotosintético y seguido por la acumulación de biomasa vegetal, o sea la producción primaria neta (PPN) (Margalef, 1983; Odum y Sarmiento, 1997). La producción primaria del fitoplancton está relacionada con la tasa fotosintética y la respiración del fitoplancton (según el método de medición se incluye la respiración del zooplancton y el bacterioplancton). La PPN varía en distintos órdenes de magnitud en los diferentes ecosistemas de la biosfera y las variaciones internas en el ecosistema son de importancia vital para la conservación de los sistemas biológicos (Knoppers, 1993; Ryszowski, 1998). Las variaciones en la PPN son determinadas por factores condicionantes físicos, químicos y biológicos.

La intensidad de la luz como fuente primaria de energía puede ser el principal factor limitante en ecosistemas cenagosos, dado que en el agua ésta disminuye exponencialmente con la profundidad, a lo que se suma la alta turbiedad del agua, afectando por tanto el espesor de la zona fótica donde es posible que se dé la fotosíntesis. Una respuesta del fitoplancton a bajas intensidades de luz es aumentar la concentración de pigmentos fotosintéticos, en particular la clorofila $a$, y cambiar la proporción de otros pigmentos que pueden utilizar las longitudes de luz que llegan a mayor profundidad (Margalef, 1983).

Se han realizado varias valoraciones de la producción primaria del fitoplancton en la Ciénaga Pajaral. Rondón (1991) estimó la PPN en 1,63 $\mathrm{gC} \cdot \mathrm{m}^{-2} \cdot$ día $^{-1}\left(598 \mathrm{gC} \cdot \mathrm{m}^{-2} \cdot \mathrm{anno}^{-1}\right)$, usando el método de botellas claras y oscuras. Rodríguez et al. (2009) estimaron la PPN en $4,33 \mathrm{gC} \cdot \mathrm{m}^{-2} \cdot$ día $^{-1}$ la PPN durante los meses de septiembre, octubre y noviembre. En el 2007 Granados et al. (2011) registraron en una estación central en la Ciénaga Grande de Santa Marta (CGSM) una PPN de $1,5 \mathrm{gC} \cdot \mathrm{m}^{-2} \cdot \mathrm{dí}^{-1}$. Durante el período octubre 1984-abril 1985 Hernández (1986) y Hernández (1988) registraron en la CGSM una PPN que varió entre 1,40 y $16,3 \mathrm{gC} \cdot \mathrm{m}^{-2} \cdot$ día $^{-1}$; en el mismo estudio se indica que la variación temporal y espacial de la producción primaria en la CGSM es causada principalmente por la entrada de aguas continentales y marinas, la turbidez del agua por resuspensión de sedimentos y la biomasa del fitoplancton; es importante anotar que la PPN en la CGSM fue más alta cuando la salinidad era 118 baja (Hernández y Gocke, 1990).
Con referencia a la comunidad de fitoplancton, Kucklick (1987) señaló que en las especies de fitoplancton de la CGSM predominan los tamaños de 2 a 20 micrometros (nanoplancton). En el mismo estudio las cianobacterias presentaron altas densidades durante la época de lluvias, cuando se da también una fuerte turbulencia en el cuerpo de agua; también observó una correlación negativa entre la clorofila $a$ y la transparencia, así como entre la clorofila $a$ y la salinidad. Además, este autor afirmó que las comunidades de fitoplancton estaban en una fase joven de crecimiento. En la CGSM Vidal (1995) registró 200 especies de diatomeas, 54 de dinoflagelados, 23 de cianofitas y 23 formas de clorófitas, euglenófitas, crisófitas y silicoflagelados. Durante el período marzo 1995-febrero de 1996 Mogollón (1996) observó un total de 91 especies de fitoplancton de la Ciénaga Pajaral, correspondiendo las mayores densidades a las cianofitas y las diatomeas, seguidas por las clorófitas y las euglenófitas.

Las especies observadas por Mogollón (1996) son típicas de hábitat de agua dulce y estuarina. Entre las abundantes se destacaron Anabaena flos-aque, Microcystis cf. robusta, Paralia sulcata, Cyclotella meneguiniana, Cyclotella glomerata, Actinocyclus normanii, Skeletonema costatum, Navicula sp., Nitzschia closterium y Plagiotopis lepidoptera, (características de hábitat de agua dulce). Durante el período octubre 88-septiembre 89 Rondón (1991) observó 34 taxa en la Ciénaga Pajaral, 8 cianofitas, 7 clorófitas, 17 diatomeas, 2 euglenófitas y 8 sin identificar. Como resultado de un estudio sobre la flora fitoplanctónica de la Ciénaga Pajaral realizado entre marzo-96 y febrero-97, Ruiz (1999) reportó 219 taxa: 73 diatomeas, 31 cianofitas, 70 clorófitas, 35 euglenófitas y 10 dinoflagelados. Este mismo autor destacó que las cianofitas fueron el grupo más abundante (66\%), luego las diatomeas ( $20 \%$ y las clorófitas (11 \%); en dicho estudio se indicó también que diez especies constituyeron el $91 \%$ de la abundancia total y los tamaños de la mayoría de las especies estuvieron entre 4,5 y $50 \mu \mathrm{m}$; además, los géneros más abundantes fueron: Oscillatoria, Synechocystis, Anabaenopsis, Nitzschia, Navicula y Ankistrodesmus.

A partir de muestreos efectuados en la CGSM entre octubre de 1999 y julio de 2000, Vidal et al. (2001) encontraron 84 taxa (14\% clorófitas, $9 \%$ cianofitas, 15 $\%$ diatomeas céntricas, $46 \%$ diatomeas pennadas, $4 \%$ dinoflagelados, $10 \%$ euglenófitas y $1 \%$ otros: criptófitas y 2 sin identificar). El $24 \%$ de las especies fueron de origen marino, el $35 \%$ dulceacuícola, el $13 \%$ estuarinas y el restante $28 \%$ sin hábitat identificado. Vidal (2010) 
recopiló toda la información disponible y presentó la iconografía y sistemática del fitoplancton en la CGSM y zonas aledañas: un total de 578 taxa (71 cianofitas, 95 diatomeas céntricas, 189 diatomeas pennadas, 59 dinoflagelados, 63 clorófitas, 8 euglenófitas y 18 no identificadas).

La situación climática en la época del presente estudio se describe con base en el informe del IDEAM (2005). Durante el periodo diciembre 1998 a febrero de 2000 (época en que se realizó el trabajo de campo) se presentó el evento climático denominado fenómeno ENSO, el cual en su fase fría, conocida como "La Niña" (15 meses de duración), fue fuerte a moderado (Velásquez y Rosales, 2002). La Niña se caracteriza por un régimen de vientos alisios fuertes desde el este, siendo típico observar condiciones más húmedas respecto a lo normal sobre la región Caribe colombiana. En mayo de 1998 finalizó El Niño, uno de los más intensos del siglo XX, y se inició La Niña. Durante el tiempo que duró el presente trabajo, el complejo lagunar del delta exterior del río Magdalena recibió grandes volúmenes de agua por desbordamiento del río Magdalena y por escorrentía, con el consiguiente lavado de los suelos de la cuenca de la Ciénaga de Pajaral.

El problema objeto de la presente investigación se planteó en términos de icuáles son los efectos de la entrada de agua dulce procedente del río Magdalena, a través del caño Aguas Negras, sobre la producción primaria de las comunidades del fitoplancton de la Ciénaga Pajaral? El estudio se basó en la hipótesis de que la entrada de agua procedente del río Magdalena, rica en nutrientes, además de reducir la salinidad del humedal (que en el pasado se había salinizado), causa un incremento en la producción primaria del fitoplancton en la Ciénaga Pajaral, y por tanto aumenta la eutrofización del sistema.

\section{ÁREA DE ESTUDIO}

La Ciénaga Pajaral se localiza en la reserva de la biosfera del complejo lagunar Ciénaga Grande de Santa Marta (CLCGSM), donde hay dos zonas de manejo especial: El Vía Parque Nacional Isla de Salamanca (al norte) y el Santuario de Fauna y Flora CGSM (al sur). La Ciénaga Pajaral hace parte de la llanura deltaica derecha del río Magdalena y de la plataforma continental del Caribe colombiano; la zona es plana y está formada por materiales aluviales y lacustres del cuaternario. El área que cubre el CLCGSM se estima en $4900 \mathrm{~km}^{2}$, de los cuales $1300 \mathrm{~km}^{2}$ son cuerpos de agua permanentes,
$730 \mathrm{~km} 2$ son ciénagas y caños y $570 \mathrm{~km}^{2}$ son de área marina (Bernal y Betancur, 1996). El complejo lagunar se localiza entre los $74^{\circ} 27^{\prime}$ y $74^{\circ} 45^{\prime}$ E y los $10^{\circ} 44^{\prime}$ y $11^{\circ} 00^{\prime} \mathrm{N}$ (Figura 1), ocupando parte de la gran llanura de inundación y acumulación formada por la divagación del río Magdalena (IGAC, 1993). La Ciénaga Pajaral se comunica con la CGSM por el caño Grande; tiene un área superficial aproximada de $44,95 \mathrm{~km}^{2}$, el volumen de agua se estima en $564 \times 106 \mathrm{~m}^{3}$ y el nivel de la columna de agua es bajo, oscilando entre $0,2 \mathrm{y}$ 1,3 m (Bernal, 1996).

La región se encuentra dentro de la zona de altas presiones atmosféricas, donde convergen los vientos alisios para formar la zona de confluencia intertropical (ZCI). Los vientos alisios del NE son el factor macroclimático condicionante para la región costera caribeña. Estos vientos dominan desde finales de noviembre hasta mediados de marzo (determinan la época seca). El Instituto Geográfico Agustín Codazzi reporta que toda la región está caracterizada por un déficit hídrico (600 a $1400 \mathrm{~mm}$ año ${ }^{-1}$, ya que siempre la evapotranspiración (aproximadamente $1800 \mathrm{~mm}$ año ${ }^{-1}$ ) es superior a la precipitación, que está alrededor de 400 a 1200 $\mathrm{mm} \cdot \mathrm{año} \mathrm{o}^{-1}$ (Lecarpentier et al., 1975), déficit que favorece la salinización de las aguas.

El Proyecto de Rehabilitación de la Ciénaga Grande de Santa Marta realizó la conexión entre el río Magdalena y el complejo lagunar mediante el dragado de los caños Clarín (dragado en 1996) y Salado, Renegado, Aguas Negras (dragados en 1998) y, a menor escala, los caños Flechas, Burro y Agua Viva (RAMSAR et al., 2004). El caño que tiene mayor influencia en la zona de estudio es Aguas Negras; se inicia en el río Magdalena, al sur de la población de Remolino, y se dirige en dirección noreste para desembocar en la Ciénaga Pajaral, siguiendo un recorrido aproximado de $28 \mathrm{~km}$. En el km $15+300$ metros se presenta el primer ramal de desviación por el caño Las Fuentes $(2 \mathrm{~km}+490 \mathrm{~m})$, que es el ramal que desemboca en la Ciénaga Pajaral. El caño Aguas Negras continua en dirección norte y en un segundo ramal se une al caño El Tambor en el kilómetro $21+100$ metros y finalmente se presenta otra derivación, el caño Guayacán $(2 \mathrm{~km}+380 \mathrm{~m})$, que se comunica con la ciénaga de Mendegua (PROCIENAGA, 1994). Con la reapertura de los caños Clarín, Aguas Negras y Renegado, se logró una reducción significativa de la salinidad, tanto en la columna de agua como en el agua intersticial del suelo. En estas zonas, antes dominadas por manglar (principalmente Avicennia 
germinans y Rhizophora mangle), en el momento de este estudio predominó el playón salino, con parches dispersos de Batis marítima, Sporobolus virginicus y Sesuvium portulacastrun. En el caño Aguas Negras y en la desembocadura del ramal Las Fuentes se encontraron Ipomoea reptans, Pontederia sp., Pistia stratiotes, Eicchornia crassipes y Neptunia postrata. Estos cambios en la salinidad se manifiestan en los procesos de regeneración natural y de desarrollo de la vegetación de agua dulce y halófita, que para la época del estudio se estaba dando en varias áreas que habían perdido totalmente la cobertura vegetal por la muerte del manglar (Botero y Mancera, 1996). Por tanto, estos cambios se asocian a la reducción de la salinidad en todo el complejo de ciénagas y caños. La disminución en los valores de salinidad también favoreció la dispersión de especies de agua dulce como Typha domingensis, la cual fue extendiendo su distribución desde el km 13 en el caño Clarín hasta sectores en donde se inició el deterioro de los bosques de manglar (La Luna y Bristol). También se observaron algunas plantas en la zona de caño Aguas Negras. En estos sitios, tanto el hidroperíodo como el micro-relieve han jugado un papel importante en el asentamiento y dispersión de T. domingensis, estableciendo una competencia por el espacio con las plántulas de manglar (INVEMAR, 2000 y observación personal) (Figura 1).

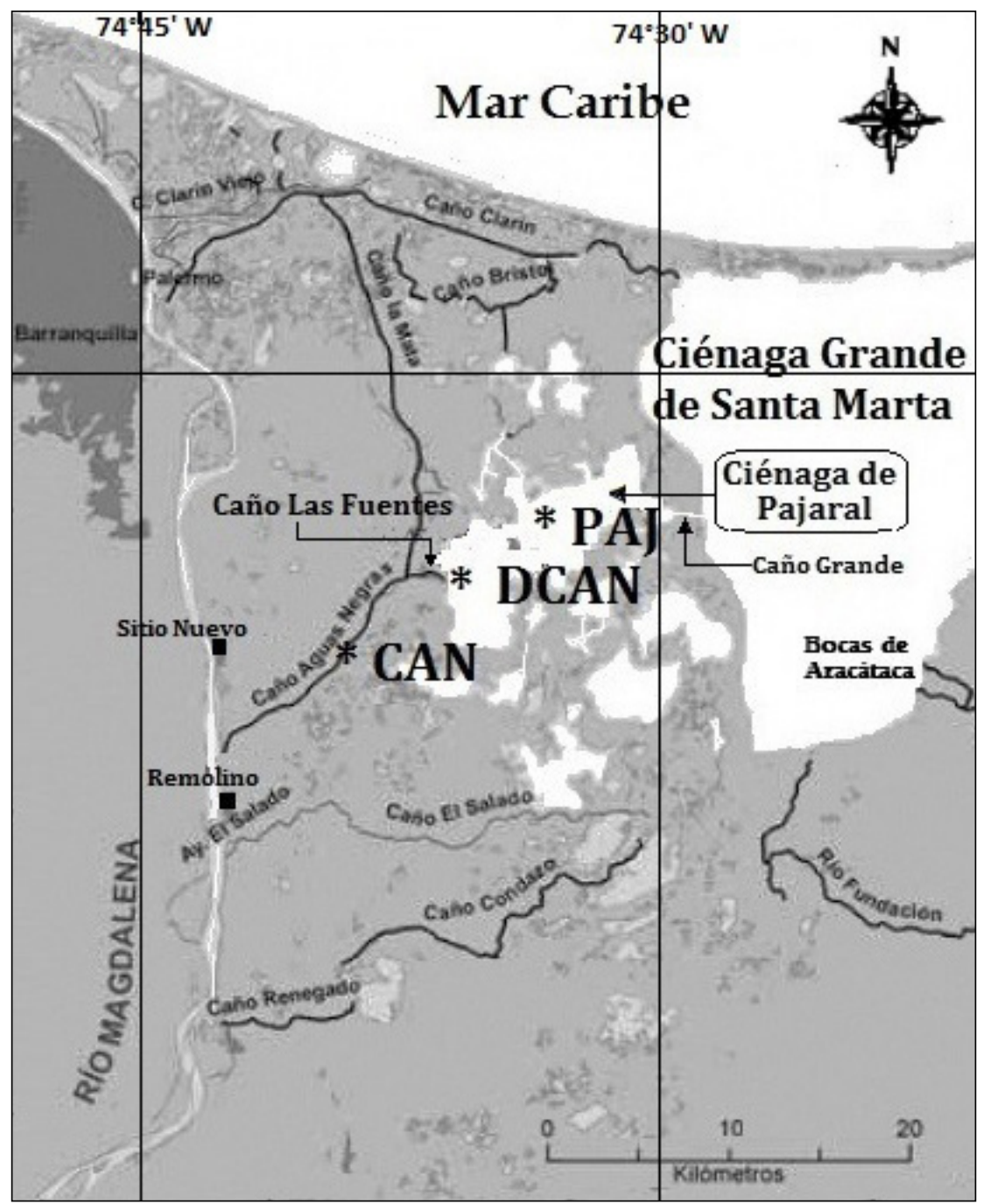

Figura 1. Localización de las estaciones de muestreo donde se valoró la producción primaria del fitoplancton en el complejo de Pajaral CAN, DCAN y PAJ. En el norte se encuentra la Vía Parque Nacional Isla de Salamanca y caño Clarín y al oeste del río Magdalena y nacimiento de Caño Aguas Negras (modificada de la Imagen SPOT). 


\section{MATERIALES Y MÉTODOS}

Las muestras se tomaron en tres estaciones: caño Aguas Negras (CAN, 1046’37” y 7439'07” W), desembocadura del caño Aguas Negras en la Ciénaga Pajaral (caño Las Fuentes) (DCAN, $10^{\circ} 48^{\prime} 51^{\prime \prime}$ y $74^{\circ} 36^{\prime} 37^{\prime \prime}$ W) y Ciénaga Pajaral (PAJ, 1049'59" y $74^{\circ} 34^{\prime} 28^{\prime \prime}$ W). En el palafito de Nueva Venecia se estableció la estación donde se realizaron los monitoreos en periodos de 48 horas $\left(10^{\circ} 49^{\prime} 59^{\prime \prime}\right.$ y $\left.74^{\circ} 34^{\prime} 28^{\prime \prime} \mathrm{W}\right)$. Para tomar las muestras de agua se utilizó una botella fabricada con un tubo de PVC sanitario de $12 \mathrm{~cm}$ de diámetro y $2 \mathrm{~m}$ de longitud, con una válvula en un extremo que permitía cerrar herméticamente y recoger la muestra de la columna de agua. El contenido de agua se vertía en un tanque plástico con capacidad de $30 \mathrm{l}$ y se repetía la toma de muestra hasta llenarlo. De este volumen de agua se tomaban cuatro submuestras de $500 \mathrm{ml}$ para las diferentes valoraciones: A) fitoplancton, B) clorofila $a \mathrm{y}$ feofitina $a, \mathrm{C}$ ) conductividad y nutrientes y D) sólidos y seston. Estos tanques se guardaban en neveras refrigeradas con hielo para ser transportadas al laboratorio, en tanto que el agua restante se usaba para valorar la producción primaria fitoplanctónica.

Para valorar la producción primaria del fitoplancton se recurrió al método de botellas claras y oscuras (Gaarder y Gran, 1927). Se homogenizaba la muestra agitando suavemente el contenido de la caneca de 30 l. Para llenar la batería (para cada estación) de 14 botellas (tipo DBO de $\pm 115 \mathrm{ml}$ de capacidad) más dos botellas tipo DBO de $500 \mathrm{ml}$ (con la que valoró la respiración en un periodo de 24 horas), se utilizaron cuatro mangueras de plástico que permitían llenar las botellas y dejar rebosar un volumen igual al volumen de éstas antes de ser tapadas.

Las incubaciones se realizaron entre las 08:30 y las 13:30 (5 horas que corresponden al segundo y tercer quinto de las horas luz, tiempo suficiente para obtener un valor promedio de la producción de un día). Los valores de oxígeno se expresaron en $\mathrm{mgO}_{2} \bullet \mathrm{l}^{-1}$. Para convertir $\mathrm{mgO}_{2}$ a gC (carbono), el valor de oxígeno en mg se multiplicó por el factor 0,313 (Grasshoff et al., 1983). Otros autores (Vollenweider, 1974; González y Berdayes, 1981) utilizan el factor 0,375 .

Las botellas claras (dos para cada nivel de intensidad de luz) se colocaron a cinco profundidades: $100 \%, 50$ $\%, 25 \%, 10 \%$ y $1 \%$ de intensidad de la luz que llega a la superficie de la masa de agua (I\%) (Figura 2). Las botellas oscuras se suspendieron en la columna de agua, a $10 \mathrm{~cm}$ del fondo. Estas botellas se colocaron dentro de un tubo de PVC con tapas, para evitar la entrada de luz. La altura de la columna de agua osciló entre 0,63 m y $1,14 \mathrm{~m}(0,89 \mathrm{~m}$ en promedio). La profundidad de incubación (PI) se calculó con base en la siguiente ecuación: PI $=$ PS• $(2,70104-[1,353325 \bullet \log (\mathrm{I} \%$ de luz deseada)], donde PI es la profundidad de incubación, PS es la profundidad de visibilidad del disco Secchi, 2,70104 es el coeficiente de extinción y 1,353325 es la constante obtenida mediante la regresión de las variables profundidad e intensidad de luz en la columna de agua en la Ciénaga de Pajaral (medida con un cuantómetro Li-cor).

Se valoró la concentración de oxígeno mediante el método de Winkler. La cantidad de oxígeno se estimó como el promedio de los valores de la pareja de botellas en cada profundidad. Luego se calculó la producción por capa, posteriormente por unidad de volumen $\left(\mathrm{m}^{3}\right)$ y finalmente por unidad de área $\left(\mathrm{m}^{2}\right)$. Este valor, obtenido en cinco horas de incubación, se calculó por día $\left(\mathrm{gC} \bullet \mathrm{m}^{-2} \bullet\right.$ día $\left.^{-1}\right)$, multiplicando por un factor que depende del número de horas luz por día, utilizando para ello los valores de horas luz del día en el que se realizó el bioensayo (Figura 2).

Las muestras de agua utilizadas para identificar las especies del fitoplancton se almacenaron en botellas plásticas de $600 \mathrm{ml}$ de capacidad, a las que se adicionó $5 \mathrm{ml}$ de lugol. Las muestras usadas para valorar clorofila se almacenaron en frascos de $500 \mathrm{ml}$ y se guardaron en neveras con abundante hielo para su transporte al laboratorio. Las muestras de agua para nutrientes se almacenaron en botellas de vidrio de $100 \mathrm{ml}$ (fosforo y hierro) y de plástico de $100 \mathrm{ml}$ (nitrógeno) y $600 \mathrm{ml}$ (seston y sólidos). Salinidad conductividad, pH y temperatura se midieron in situ con equipos portátiles WTW (pH-metro conductímetro). Para la valoración de nutrientes se siguió la metodología establecida en Grasshoff et al. (1983) y APHA et al. (1992).

El fitoplancton se evaluó de manera cualitativa y cuantitativa. Se identificaron 191 especies y se valoró la abundancia, con ayuda de un microscopio Nikon Eclipse 4000. Dada la alta densidad de células y de material detrítico acompañante (no se requirió concentrar la muestra), se tomaron submuestras de $50 \mu \mathrm{L}$ medidas con una pipeta Eppendorf (capacidad de $1200 \mu \mathrm{L}$ y graduación $0,1 \mu \mathrm{L}$ ). Este volumen 
se vertió en una placa portaobjeto y se colocó el cubreobjetos. Luego se valoró toda el área mediante transectos lineales con aumento de $400 \mathrm{X}$ y en caso de ser necesario $1000 \mathrm{X}$. Se observaron de 15 a 20 submuestras $(50 \mu \mathrm{L})$ de cada muestra, y se contaron el número de individuos por mililitro de muestra. Para la identificación taxonómica se utilizaron los siguientes trabajos: Yacubson (1969; 1972; 1974a; 1974b; 1980; 1984), Parra et al., (1982), Yacubson y Bravo (1982; 1986); Anagnostidis y Komárek (1988), Vidal (1995; 1996), Pereira et al. (2000) y Vidal et al. (2001).

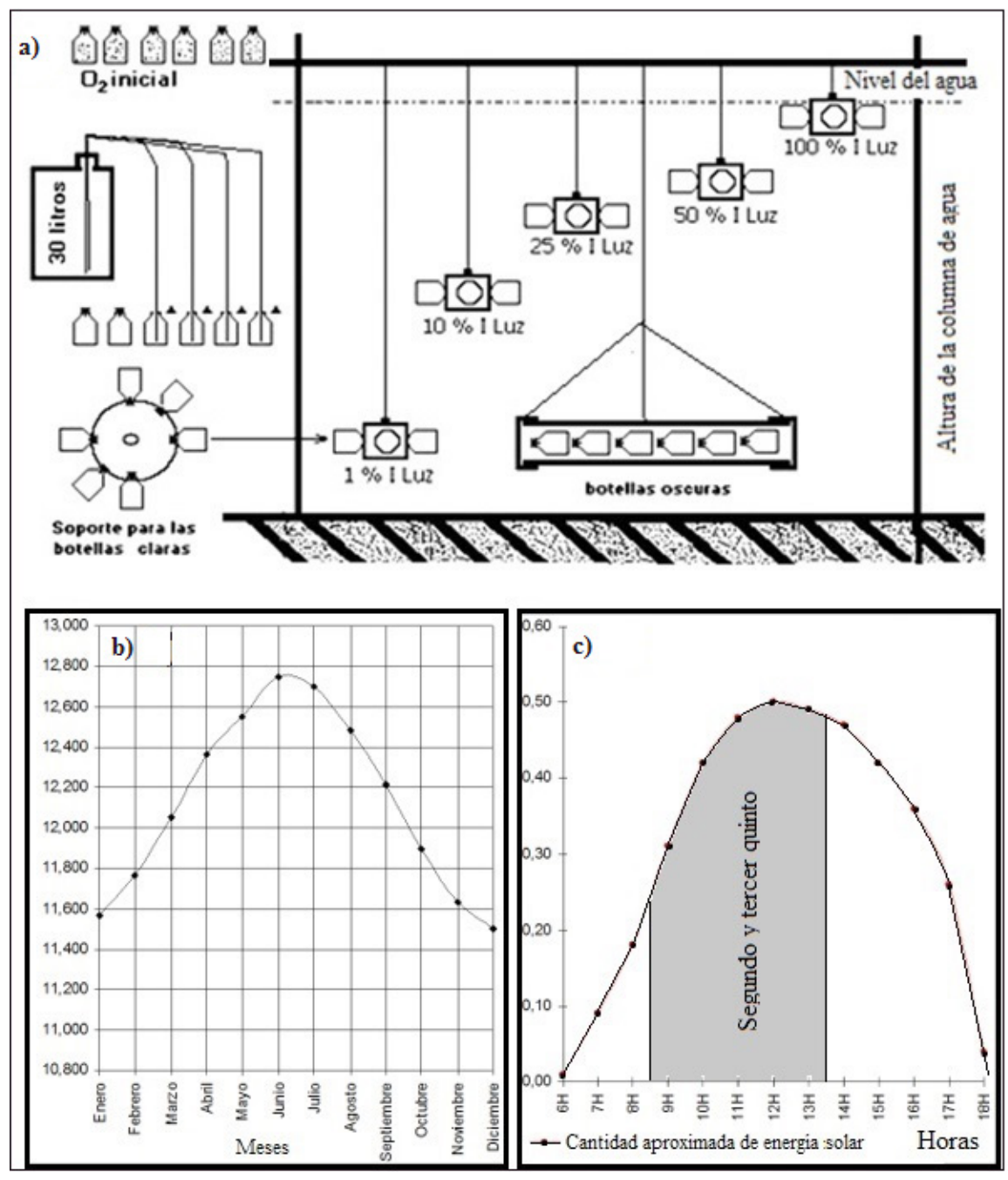

Figura 2. A) Diseño de la incubación de las botellas claras y oscuras para valorar la producción primaria del fitoplancton. B) Horas de luz promedio mes en la región de Santa Marta y C) intensidad lumínica aproximada durante un día y tiempo de medición de la producción primaria.

La extracción de la clorofila $a$ y la feofitina $a$ se realizó con acetona al $90 \%$. Se usaron filtros Whatmann GF/F $(0,7 \mu \mathrm{m})$, espectrofotómetro Bausch \& Lomb Spectronic, lampara de tungsteno y celdas de cuarzo de 1 y $5 \mathrm{~cm}$. Para el cálculo de la concentración de pigmentos se utilizaron las ecuaciones propuestas por 122 Lorenzen (1967):

$$
\begin{aligned}
& \text { Clorofila } \boldsymbol{a}\left(\mu \frac{\mathrm{g}}{\mathrm{l}}\right)=26,73 \cdot(\mathrm{D} 665 \mathrm{o}-\mathrm{D} 665 \mathrm{a}) \cdot\left[\frac{\mathrm{v}}{(\mathrm{V} \cdot \mathrm{L})}\right] \\
& \text { Feofitina } \boldsymbol{a}(\mu \mathrm{g} / \mathrm{l})=26,73 \cdot[(1,7 \cdot \mathrm{D} 665 \mathrm{a})-\mathrm{D} 665 \mathrm{o}] \cdot\left[\frac{\mathrm{v}}{(\mathrm{V} \cdot \mathrm{L})}\right]
\end{aligned}
$$

Donde:

$\mathrm{V}=$ Volumen, en litros, de muestra filtrada.

$\mathrm{V}=$ Volumen, en mililitros de acetona $90 \%$ usada para extracción. $\mathrm{L}=$ Longitud de la cubeta espectrofotométrica usada (1 y $5 \mathrm{~cm}$ ). 
D6650 = Densidad óptica a $665 \mathrm{~nm}$, corregida sin acidificar .

D665a $=$ Densidad óptica a $665 \mathrm{~nm}$, corregida acidificada .

\section{RESULTADOS Y DISCUSIÓN}

\section{Producción primaria}

Los valores de PPN, PPB y respiración se muestran en las Figuras 3, 4 y 5, respectivamente. En la estación

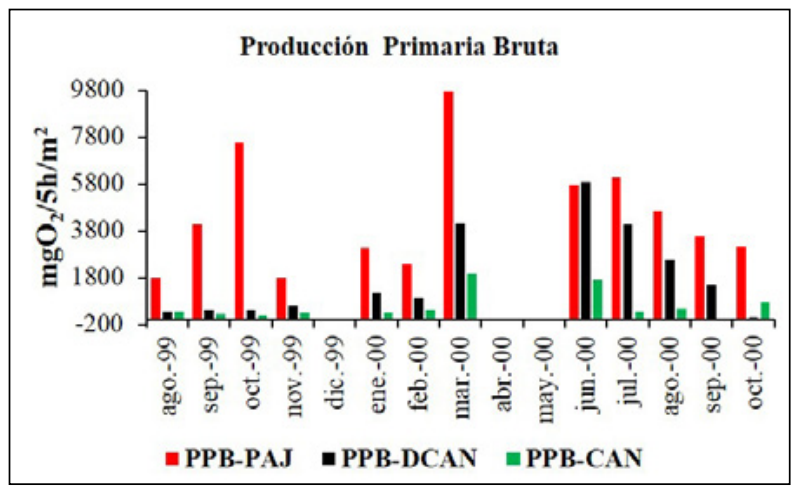

Figura 3. Producción primaria neta (PPN), $\mathrm{mgO}_{2} / 5 \mathrm{~h} / \mathrm{m}^{2}$. Estaciones caño Aguas Negras, CAN desembocadura del caño Aguas Negras DCAN y Ciénaga Pajaral PAJ.

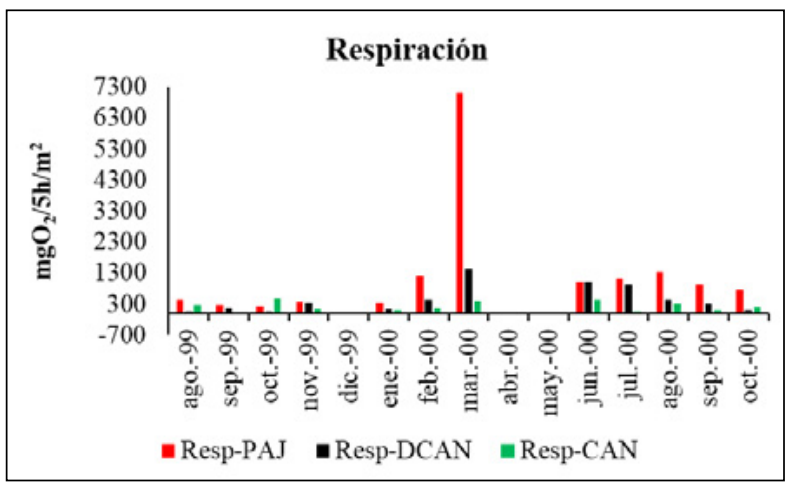

Figura 5. Salinidad en las estaciones caño Aguas Negras, Desembocadura del caño Aguas Negras y Ciénaga de Pajaral.

En octubre del 2000 se observó que la PPB en la estación DCAN fue casi igual a la respiración. Esta alta respiración no pudo ser causada solo por la respiración del fitoplancton; es posible que el bacterioplancton fuera en parte el responsable, lo que se explicaría por la baja conductividad eléctrica en uno de los meses con mayor precipitación pluvial, ocasionando que la
CAN los valores de PPN fueron relativamente bajos (son aguas del río Magdalena). En dos mediciones la PPN fue menor que la respiración. El pico de máxima producción se presentó en marzo y junio, cuando también se observaron valores altos de conductividad eléctrica (aguas salobres). Los valores de salinidad estuvieron en cero en la mayoría de las observaciones realizadas en la estación PAJ (Figura 6).

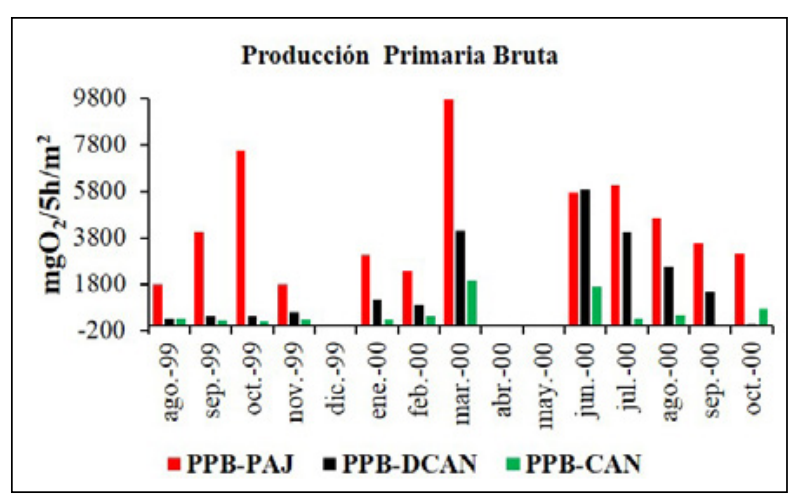

Figura 4. Producción primaria bruta (PPB), $\mathrm{mgO}_{2} / 5 \mathrm{~h} /$ $\mathrm{m}^{2}$. Estaciones caño Aguas Negras, CAN desembocadura del caño Aguas Negras DCAN y Ciénaga Pajaral PAJ.

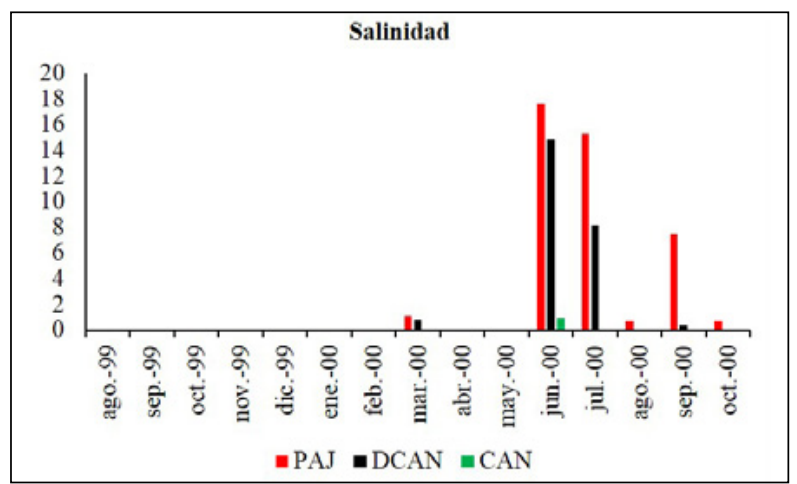

Figura 6. La respiración $(\mathrm{R}=\mathrm{PPB}$ - PPN) de la comunidad fitoplanctónica en las tres estaciones caño Aguas Negras, desembocadura del caño Aguas Negras y Ciénaga Pajaral.

entrada de agua dulce implique el transporte de una alta carga de compuestos orgánicos y, por consiguiente, una alta demanda de oxígeno. Algo similar se observó en agosto y septiembre de 1999, cuando se presentaron valores de cero en las salinidades. En junio del 2000 se presentó el pico de máxima PPN y para ese momento había aumentado la salinidad. 
En la estación PAJ, al igual que en las estaciones DCAN y CAN, la salinidad fue baja en la mayoría de los muestreos (Figura 6). Se presentaron tres picos de producción primaria, correspondientes a los muestreos realizados en sep-99, oct-99 y jun-00, y un mínimo en feb-00. Los dos primeros se presentaron cuando la salinidad fue cero (baja conductividad eléctrica) y el tercero cuando la salinidad logró su máximo valor (alta conductividad eléctrica). Esta situación fue observada en otros estudios (Hernández, 1986; Hernández, 1988; Rondón, 1991; Granados et al., 2011), lo que indicaría que el cambio de salinidad tiene un efecto en el metabolismo de las especies del fitoplancton, ya sea por cambios en la composición de especies o por modificar un factor limitante (una menor turbidez del agua).

Los valores de PPB, PPN y respiración difirieron significativamente entre las tres estaciones, lo que parece ser una respuesta al gradiente de conductividad eléctrica del agua (salinidad). Si bien el tiempo de duración de las incubaciones de las botellas claras y oscuras fue de cinco horas (08:00-13:00), los valores observados en el $100 \%$ y el $50 \%$ de intensidad de luz indican la ocurrencia del proceso de foto-respiración causada por alta intensidad luminosa. La fotorespiración se observó solo en las muestras de DCAN y PAJ, lo que implica que los valores reales de fotosíntesis fueron mayores a los estimados con base en el oxígeno disuelto de las botellas a 100 y $50 \%$ de intensidad luminosa.

Los valores de PPN en CAN oscilaron del siguiente modo: máxima de $2,95 \mathrm{gC} \cdot \mathrm{m}^{-2} \cdot$ día $^{-1}$ (jun-00) y mínima (valor negativo, donde la respiración supera la fotosíntesis) de $-0,33 \mathrm{gC} \cdot \mathrm{m}^{-2} \cdot$ día $^{-1}$ (oct-00), siendo el promedio $0,60 \pm$ $0,94 \mathrm{gC} \cdot \mathrm{m}^{-2} \cdot \mathrm{dí}^{-1}$. En DCAN el máximo valor fue 4,30 $\mathrm{gC} \cdot \mathrm{m}^{-2} \cdot$ día ${ }^{-1}$ (jun-00) y el mínimo $-0,05 \mathrm{gC} \cdot \mathrm{m}^{-2} \cdot$ día $^{-1}($ oct-00), en tanto que el promedio fue $2,57 \pm 3,05 \mathrm{gC} \cdot \mathrm{m}^{-2} \cdot \mathrm{día}^{-1}$. En PAJ el máximo fue $10,19 \mathrm{gC} \cdot \mathrm{m}^{-2} \cdot$ día $^{-1}$ (oct-99) y el mínimo $0,18 \mathrm{gC} \cdot \mathrm{m}^{-2} \cdot \mathrm{dí}^{-1}(\mathrm{jul}-00)$, mientras que el promedio fue $3,92 \pm 2,76 \mathrm{gC} \cdot \mathrm{m}^{-2} \cdot$ día $^{-1}$. Los valores máximos de PPN ocurrieron en PAJ y si bien en DCAN se dio un pico, éste comparativamente se encontró entre los valores bajos observados en PAJ. Tanto la PPB como la PNN de CAN son bajos al compararlos con las otras dos estaciones. También es notable el hecho de que se presentaron valores negativos de PPN, indicando que en tales casos la tasa respiratoria superó a la fotosíntesis neta.

El promedio de la PPB fue $3,13 \mathrm{gC} \cdot \mathrm{m}^{-2} \cdot \mathrm{d}^{-1}$. Se estimó que la PPB anual es de $1142,64 \mathrm{gC} \cdot \mathrm{m}^{-2} \mathrm{El}$ promedio de la PPN fue $2,36 \mathrm{gC} \cdot \mathrm{m}^{-2} \cdot \mathrm{d}^{-1}$, lo que significa $860,96 \mathrm{gC} \cdot \mathrm{m}^{-2} \cdot \mathrm{a}^{-1}$. El área 124 de PAJ es de $44,95 \mathrm{~km}^{2}$, por lo que la PPN se estima en

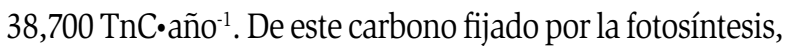
una parte permanecería en los sedimentos como materia orgánica no biodegradable, otra parte es exportada como biomasa animal de herbívoros (peces, crustáceos) y carnívoros como peces y aves piscívoras. Esta biomasa sale del sistema por pesca y migración, en tanto que otra parte de la biomasa de herbívoros se quedaría en el sistema como material detrítico depositado en sedimentos, donde se liberarían elementos nutrientes.

\section{Tasa respiratoria}

El análisis de la tasa de respiración indica que en algunos de los bioensayos la producción de oxígeno fue mayor que su consumo, por lo que la razón Producción/Respiración fue mayor que uno (1). Se presentaron casos donde la respiración fue mayor que la PPN, es decir las demandas de oxígeno superaron la cantidad de oxígeno liberado en el proceso de producción primaria. El comportamiento de los valores de respiración en las estaciones de muestreo se presenta en la Figura 5, donde se observa que los picos de tasa respiratoria se presentaron en marzo y agosto del 2000 y los mínimos en octubre 1999 y octubre 2000. Se observa una relación positiva entre el comportamiento de la conductividad eléctrica del agua (salinidad) y la tasa respiratoria. Los coeficientes de correlación para las tres estaciones de trabajo fueron: CAN, $r=0,76$; DCAN, $r=0,79$; y PAJ, $r=$ 0,51 ( $n=12$ en todos los casos). La localización de las tres estaciones permitió detectar un gradiente salino (menor a mayor) en la dirección CAN, DCAN y PAJ.

Se presentaron tasas respiratorias que superaron a la fotosíntesis neta, lo que denota un claro comportamiento heterotrófico de la comunidad planctónica. El análisis de la tasa de respiración indica que en la mayoría de las observaciones el comportamiento de la comunidad fitoplanctónica es autótrofo y, por los altos valores, se infiere un estado de eutrofización que en algunos casos es muy alto, alcanzando niveles de hipertrofia y ocurriendo bajas de oxígeno en la noche. En el mes de marzo se presentó en CAN un pico en la tasa respiratoria, cuando se da un aumento en la salinidad y la producción primaria es relativamente alta en las tres estaciones. En agosto del 2000 la tasa respiratoria en los tres puntos de muestreo es alta y corresponde a una baja de la salinidad.

La PPN estuvo correlacionada positivamente con el contenido de sales en el agua. Los coeficientes de correlación fueron los siguientes: CAN, $r=0,77(p=0,0035)$; DCAN, $r=0,84(p=0,00078)$; $y$ PAJ, $r=0,65(p=0,0229)$. 
Por otra parte, el coeficiente de correlación obtenido con todos los datos fue $r=0,742(n=36, p=<0,05)$. Estos resultados indican una aparente contradicción con otros estudios, puesto que en este caso a mayor salinidad mayor PPN (Figura 3). Una posible explicación sería que la entrada de agua dulce afecta la tasa fotosintética, en la medida que aumenta la cantidad de material inorgánico disuelto y en suspensión en el agua, lo que atenúa la penetración de la luz (reduce la capa fótica); además, el agua que entra tiene bajas densidades de fitoplancton (procede del río Magdalena) y a esto se suma la alta carga orgánica que transporta el agua del río lo que aumenta la demanda de oxígeno por parte del bacterioplancton, sin desconocer la respiración del zooplancton, en particular por organismos protistas asociados a las bacterias que constituyen su alimento (Gocke et al., 2004).

\section{Clorofila $a$ y Feofitina $a$}

Los valores de clorofila $a$ (Figuras 7 y 8 ) se situaron dentro de los valores encontrados por otras investigaciones en el área. En PAJ los valores extremos fueron $16,7 \mu \mathrm{g}^{-1} \mathrm{l}^{-1}$ en nov-99 y $174,3 \mu \mathrm{g} \cdot \mathrm{l}^{-1}$ en jul-00. Para la Ciénaga Pajaral Rondón (1991)

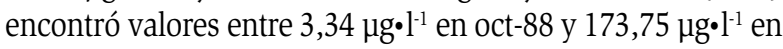
jul-89; Mogollón (1996) registró valores entre 0,58 $\mu \mathrm{g}^{\cdot \mathrm{I}^{-1}}$ en dic-95 y 212,18 $\mu \mathrm{g} \cdot \mathrm{l}^{-1}$ en feb-96. Después de la reapertura del caño Clarín que desemboca en la CGSM, Ruiz (1999) registró $7,42 \mu \mathrm{g} \cdot \mathrm{l}^{-1}$ en oct-96 y 204,11 $\mu \mathrm{g} \cdot \mathrm{l}^{-1}$ en sep-95. Mogollón (1996) coincide en afirmar que los mayores contenidos de clorofila $a$ se presentan después de los períodos de lluvia y son seguidos por un aumento en los niveles fluviométricos en el sistema estuarino, durante el período de aguas altas, cuando se presentaron importantes precipitaciones en la región acompañadas de un aumento en el flujo de agua proveniente del río Magdalena cargado de nutrientes inorgánicos y materia orgánica disuelta y particulada.

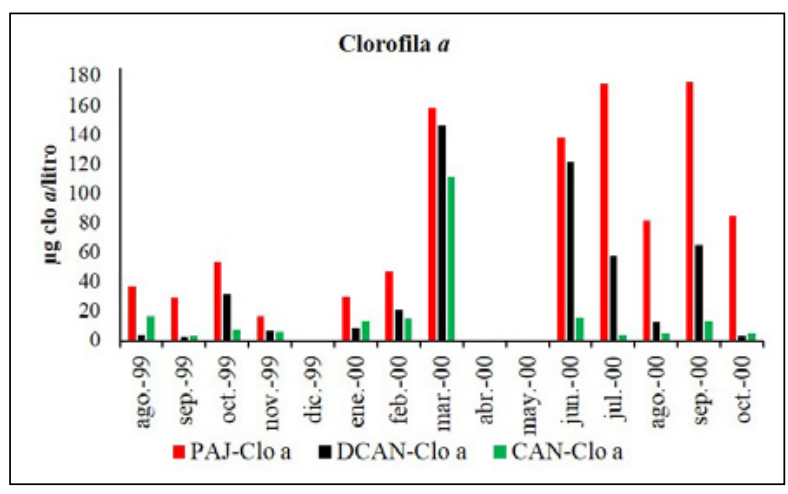

Figura 7. Concentración de clorofila $a$ en las estaciones caño Aguas Negras, CAN desembocadura del caño Aguas Negras DCAN y Ciénaga Pajaral PAJ.
La feofitina $a$ corresponde a la porción de clorofila $a$ degradada (Andreo y Vallejo, 1984). En el presente estudio se observó que la concentración de clorofila $a$ en PAJ fue mayor que la de feofitina $a$, con una excepción en febrero de 2000, cuando la concentración de feofitina $a$ fue mayor, coincidiendo con una densidad de fitoplancton y una PPN relativamente bajas. Los valores de feofitina $a$ en PAJ se situaron en el rango $2,9 \mu \mathrm{g} \cdot \mathrm{l}^{-1}$ en ago-00 y 86,4 $\mu \mathrm{g} \cdot \mathrm{l}^{-1}$ en feb-00. En DCAN la concentración de feofitina $a$ fue mayor en siete muestreos a lo observado en PAJ. Dichos valores fueron $3,2 \mu \mathrm{g} \cdot \mathrm{l}^{-1}$ en sep-00 y $41,4 \mu \mathrm{g} \cdot \mathrm{l}^{-1}$ en jun-00. En CAN la concentración de feofitina $a$ fue mayor a la de PAJ en seis muestreos, con valores entre $0,8 \mu \mathrm{g} \cdot \mathrm{l}^{-1}$

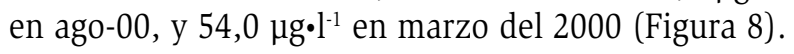

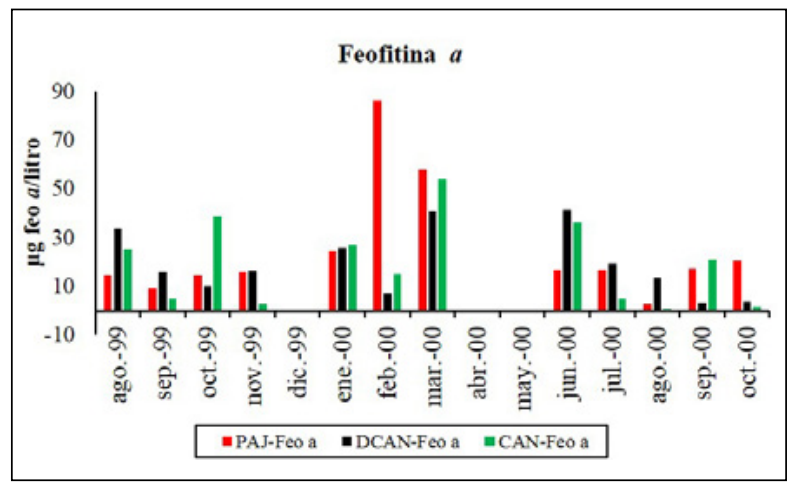

Figura 8. Concentración de feofitina $a$ en las estaciones caño Aguas Negras, CAN desembocadura del caño Aguas Negras DCAN y Ciénaga Pajaral PAJ.

\section{Densidad del fitoplancton}

En este estudio se observaron 191 taxones distribuidos de la siguiente forma: 45 cianófitas, 10 diatomeas céntricas, 47 diatomeas pennadas, 3 dinoflagelados, 62 clorófitas, 19 euglenófitas y 6 formas flageladas. En la mayoría de las muestras el fitoplancton fue característico de aguas dulces. El aumento en la salinidad modificó la composición y la densidad de especies, evidenciando que la comunidad fitoplanctónica respondió rápidamente a los cambios de salinidad asociados a otros cambios en variables físicas y químicas (turbidez, concentración de nutrientes). En PAJ se identificaron 180 especies, en DCAN 161 y en CAN 160. Se observó una correlación significativa positiva entre la concentración de clorofila $a$ y la densidad (células $\cdot \mathrm{ml}^{-1}$ ), que se calculó con base en datos obtenidos de las 36 muestras $(r=0,7064, p=$ 0,000001). También fue significativa la correlación entre clorofila $a$ y conductividad eléctrica (salinidad): CAN, $\mathrm{r}$ $=0,64(\mathrm{p}<0,0500)$; DCAN, $\mathrm{r}=0,71(\mathrm{p}<0,0500)$; $\mathrm{PAJ}$, $\mathrm{r}=0,76(\mathrm{p}<0,0500)$ (Figura 9). 


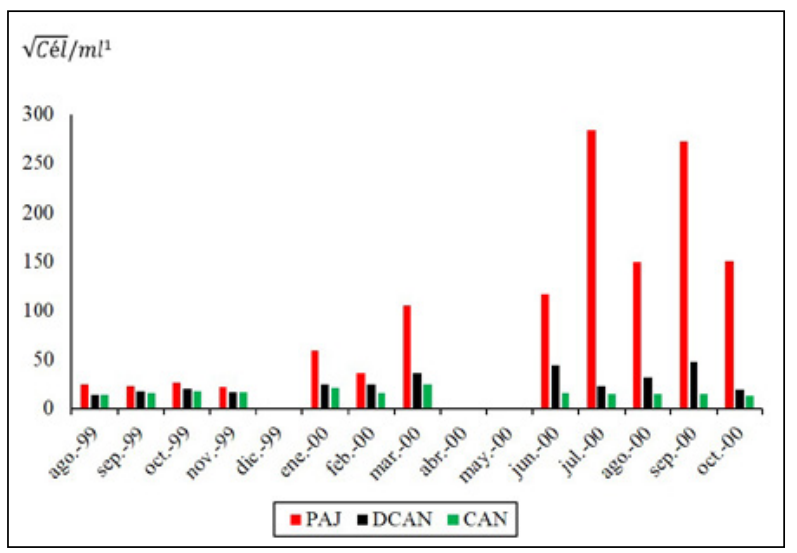

Figura 9. Densidad de fitoplancton por muestreo en las estaciones caño Aguas Negras, CAN desembocadura del caño Aguas Negras DCAN y Ciénaga Pajaral PAJ.

Las cianofitas contribuyeron con el 23,6 \% del total de especies, predominando las formas filamentosas (tipo Oscillatoria), las esféricas (tipo Chroococcus) y las láminas (tipo Merismopedia). En CAN se encontraron 33 especies de cianofitas, en DCAN 41 especies y en PAJ 45 especies. Por otra parte, de las 45 especies de cianofitas identificadas, seis presentaron altas densidades y alta frecuencia (comunes a las tres estaciones de muestreo). Sus abundancias (células $\cdot \mathrm{ml}^{-1}$ ) aportaron la mayor cantidad de células en los diferentes muestreos. Este grupo aportó la mayor abundancia de células en todas las estaciones. Por su pequeño tamaño, las especies de cianofitas se ubicaron en la categoría de nanoplancton (2-20 $\mu \mathrm{m})$ y unas pocas en la categoría de microplancton

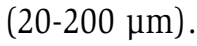

En cuanto a las diatomeas céntricas, se identificaron 10 taxones $(5,2 \%)$. Las densidades más altas correspondieron a las especies Actynocyclus normanii, Coscinodiscus cf. gigas y Cyclotella meneghiniana. Las especies observadas están en las categorías de nanoplancton y microplancton. Se identificaron 47 especies de diatomeas pennadas $(24,6 \%)$; las densidades mayores correspondieron a los géneros Navicula y Nitzschia. También se ubicaron en las categorías de nanoplancton y microplancton. Los dinoflagelados observados fueron tres taxones $(1,6$ $\%$ ), siendo Alexandrium minutun la especie más importante. La frecuencia y el número de individuos de este grupo fueron bajos, significando una baja contribución al fitoplancton en la zona de estudio. En lo que respecta a las clorófitas (62 taxa, 32,5 \%), los géneros Oocystis, Scenedesmus y Eudorina 126 presentaron una densidad alta y aparecieron en la mayoría de las muestras (categorías nanoplancton y microplancton).

En el grupo de las euglenófitas (19 taxones, 9,9 \%) se destacaron por su mayor frecuencia las especies de los géneros Euglena (9), Phacus (5) y Trachelomonas (5). En este último género, la especie más abundante y frecuente fue Trachelomona armada. Estos géneros se ubican en las categorías de nanoplancton y microplancton. En el grupo de las especies flageladas se identificaron cinco taxones $(2,6 \%)$, uno de los cuales fue asignado a las criptófitas, en tanto que los otros cuatro fueron especies flageladas no identificadas; todas estas se ubicaron en la categoría del nanoplancton, con tamaños entre 0,5 y $10 \mu \mathrm{m}$.

La eutrofización evidenciada en la Ciénaga Pajaral se explica por el incremento de los niveles de nutrientes, principalmente nitrógeno y fósforo (Tabla 1); el origen de éstos se explicaría por los aportes difusos de aguas provenientes del lavado de suelos de áreas de cultivos y ganadería que se encuentran en los terrenos que separan el complejo de humedales y el río Magdalena, los nutrientes que transportan las aguas del río Magdalena, el lavado de suelos sin vegetación (manglar muerto y orillas del caño sin cobertura vegetal) que aportan minerales al agua, además de la acumulación de sedimentos ricos en elementos nutrientes y los aportes puntuales de aguas residuales domésticas no tratadas, vertidas directamente al sistema acuático. El exceso de nutrientes y en especial de fósforo es favorable para las cianobacterias, que se benefician de las altas temperaturas (mayores de 25 ${ }^{\circ} \mathrm{C}$ ), la baja turbulencia del agua por la disminución de vientos en la época de lluvias y la intensidad luminosa de la región de Santa Marta, que recibe entre 5,5 y $6,0 \mathrm{kWh} / \mathrm{m}^{2}$ de radiación solar por día (IDEAM, 2005).

\section{CONCLUSIONES}

Como se esperaba, la producción primaria de la comunidad fitoplanctónica fue más alta en la estación ubicada en la Ciénaga Pajaral y más baja en las aguas del caño Aguas Negras.

La tasa de respiración fue mayor en la Ciénaga Pajaral comparada con las otras dos estaciones, dado que la comunidad fitoplanctónica en Pajaral se encuentra en un estado de mayor madurez (densidad alta y relaciones tróficas más complejas). Además, la mayor 
tasa de respiración se puede atribuir parcialmente al metabolismo del bacterioplancton.

La mayor PPN ocurrió en la estación PAJ, oscilando entre 0,2 gC $\cdot \mathrm{m}^{-2} \cdot$ día $^{-1}$ y $10,2 \mathrm{gC} \cdot \mathrm{m}^{-2} \cdot$ día $^{-1}$ (promedio de 3,9 $\pm 2,8$ $\left.\mathrm{gC} \cdot \mathrm{m}^{-2} \cdot \mathrm{dí}^{-1}\right)$. Si bien las tasas fotosintéticas fueron altas, éstas se dan en una delgada capa (zona fótica) dada la alta turbidez y la poca profundidad de los cuerpos de agua.
Los valores de PPN en la Ciénaga Pajaral son comparables a los observados en la Ciénaga Grande de Santa Marta por Hernández y Gocke (1990), quienes registraron un valor máximo de $16,3 \mathrm{gC} \cdot \mathrm{m}^{-2} \cdot \mathrm{d}^{-1}$.

Los altos valores de PPN, densidad de fitoplancton, concentración de clorofila $a$ y turbidez del agua indican que la Ciénaga Pajaral está en un nivel alto de eutrofización.

Tabla 1. Valores de concentración de Ortofosfato $\left(\mathrm{PO}_{4}\right)$, Nitrógeno inorgánico total $(\mathrm{Nt})$, y bióxido de carbono $\left(\mathrm{CO}_{2}\right)$ en las estaciones de Ciénaga Pajaral (PAJ), desembocadura del caño Aguas Negras (DCAN) y Caño Aguas Negras (CAN), durante el período agosto 1999 a octubre 2000.

\begin{tabular}{|c|c|c|c|c|c|c|c|c|c|}
\hline & PAJ & DCAN & CAN & PAJ & DCAN & CAN & PAJ & DCAN & CAN \\
\hline Fechas & $\begin{array}{c}\mathrm{PO}_{4} \\
\left(\mathrm{mg}^{-1} \mathbf{l}^{-1}\right)\end{array}$ & $\begin{array}{c}\mathrm{PO}_{4} \\
\left(\mathrm{mg}^{-1} \mathbf{l}^{-1}\right)\end{array}$ & $\begin{array}{c}\mathrm{PO}_{4} \\
\left(\mathrm{mg}^{-1} \mathbf{l}^{-1}\right)\end{array}$ & $\begin{array}{c}\mathrm{Nt} \\
\left(\mathrm{mg} \cdot \mathbf{l}^{-1}\right)\end{array}$ & $\begin{array}{c}\mathrm{Nt} \\
\left(\mathrm{mg} \cdot \mathbf{l}^{-1}\right)\end{array}$ & $\begin{array}{c}\mathrm{Nt} \\
\left(\mathbf{m g} \bullet \mathbf{l}^{-1}\right)\end{array}$ & $\begin{array}{c}\mathrm{CO}_{2} \\
\left(\mathrm{mg}^{-1} \mathrm{l}^{-1}\right)\end{array}$ & $\underset{\left(\mathrm{mg}^{2} \mathrm{l}^{-1}\right)}{\mathrm{CO}}$ & $\begin{array}{c}\mathrm{CO}_{2} \\
\left(\mathrm{mg}^{-1} \mathrm{I}^{-1}\right)\end{array}$ \\
\hline ago-99 & 0,30 & 0,60 & 0,50 & 0,62 & 1,04 & 1,18 & 0,19 & 0,19 & 0,16 \\
\hline sep-99 & 0,02 & 0,10 & 0,10 & 0,38 & 1,05 & 0,95 & 0,09 & 0,12 & 0,02 \\
\hline oct-00 & 1,10 & 1,50 & 1,50 & 0,70 & 0,66 & 1,09 & 0,47 & 0,08 & 0,03 \\
\hline nov-99 & 0,10 & 0,30 & 0,20 & 0,22 & 2,49 & 0,21 & 0,27 & 0,38 & 0,23 \\
\hline ene-00 & 0,50 & 0,80 & 0,80 & 9,00 & 1,23 & 1,33 & 0,34 & 0,21 & 0,09 \\
\hline feb-00 & 0,20 & 0,20 & 0,40 & 2,87 & 0,63 & 2,38 & 0,21 & 0,09 & 0,08 \\
\hline mar-00 & 0,40 & 0,30 & 0,30 & 1,21 & 1,24 & 1,27 & 0,10 & 0,01 & 0,02 \\
\hline jun-00 & 1,20 & 1,40 & 0,70 & 3,74 & 3,43 & 1,54 & 0,14 & 0,06 & 0,04 \\
\hline jul-00 & 0,20 & 0,10 & 0,10 & 1,26 & 1,14 & 0,97 & 0,27 & 0,14 & 0,06 \\
\hline ago-00 & 9,50 & 7,20 & 4,70 & 1,19 & 1,13 & 0,98 & 0,17 & 0,07 & 0,03 \\
\hline sep-00 & 6,70 & 13,10 & 16,07 & 0,27 & 1,00 & 1,39 & 0,12 & 0,05 & 0,02 \\
\hline oct- 00 & 5,00 & 5,60 & 4,10 & 0,32 & 0,58 & 0,54 & 0,27 & 0,23 & 0,02 \\
\hline
\end{tabular}

El estado trófico de la Ciénaga Pajaral está determinado por la entrada de nutrientes y materia orgánica transportada por las aguas del río Magdalena (y su desbordamiento en época de lluvias), determinando una acumulación progresiva de nutrientes y materia orgánica en los sedimentos; a este proceso de eutroficación contribuye también el hecho de ser un cuerpo de agua somero, con profundidades de $20 \mathrm{a}$ $130 \mathrm{~cm}$, lo que facilita la resuspensión de sedimentos y nutrientes, y un espejo de agua de 4,495 hectáreas, que facilita la mezcla de las aguas por la acción del viento.

La producción primaria de los cuerpos de agua en general y, en particular, de los que han sido objeto de este estudio, con valores de PPN del orden de los $10,19 \mathrm{gC} \cdot \mathrm{m}^{-2} \cdot \mathrm{dí}^{-1}$, permite comprender la función del fitoplancton como una bomba de carbono que reduce el $\mathrm{CO}_{2}$ agua-atmósfera. En la región, el área de los cuerpos de agua permanentes se estima en $1,300 \mathrm{~km}^{2}$; con base en lo anterior, el valor de $\mathrm{CO}_{2}$ fijado podría ser del orden de los 13,247,000,000 gC•1,300 km²-2ía ${ }^{-1}$.

En los meses de julio, agosto y septiembre del 2000 se presentó un florecimiento de cianofitas, correspondiendo las mayores densidades a Anabaenopsis circularis, Oscillatoria sp. y Lyngbya limnetica. Predominaron varios géneros de cianofitas que son potencialmente liberadores de sustancias toxicas, como son Anabaena, Anabaenopsis, Aphanizomenon, Lyngbya y Microcystis. El segundo grupo en importancia fueron las diatomeas pennadas, cuyas mayores densidades correspondieron a los géneros Navicula y Nitzschia. 
El efecto del evento climático fenómeno ENSO en su fase fría causó abundantes entradas de agua dulce tanto del rio Magdalena como de la escorrentía local por lluvias; esto determinó bajas en la salinidad en la Ciénaga Pajaral y aportes de sedimentos inorgánicos, elementos nutrientes y materia orgánica, lo que causó una alta turbiedad del agua y limitó la zona fótica (en la mayoría de las observaciones no llegó suficiente luz al fondo).

La reapertura del caño Aguas Negras afectó la concentración de sales, en la medida que se da una recuperación de la cobertura vegetal y la presencia de vegetación acuática; por otra parte, aumentó la carga de sedimentos que entra al sistema contribuyendo a la colmatación de los humedales, como se observó en la desembocadura del caño en la Ciénaga Pajaral (DCAN) donde se formó un mini delta por acumulación de sedimentos, dificultando la navegación y la entrada al caño Las Fuentes.

\section{AGRADECIMIENTOS}

La información presentada en este documento hace parte del Proyecto "Monitoreo de los procesos indicadores de recuperación de ecosistemas en el delta exterior derecho del río Magdalena", financiado por la Universidad del Magdalena, COLCIENCIAS, GTZ, CORPAMAG y UAESPNN. Código 1117-09-040-98 (Contrato 361-98). El trabajo de campo fue apoyado por habitantes del palafito de Nueva Venecia (complejo Pajarales).

\section{BIBLIOGRAFÍA}

Anagnostidis, K. y Komárek, J. 1988. Modern approach to the classification system of cyanophytes. 3. Oscillatoriales. Archiv fuer Hydrobiologie Supplement 80 (1-4): 327-472.

Andreo, C. y Vallejo, R. 1984. Fotosíntesis. Secretaria General de la Organización de Estados Americanos, Washington.

American Public Health Association APHA, American Water Works Association AWWA, and Water Pollution Control Federation WPCF. 1992. Standard methods for the examination of water and wastewater. American Public Health Association.S.A, Washington.

Bernal, F. G. 1996. Caracterización geomorfológica de la llanura deltaica del río Magdalena con énfasis en el sistema lagunar de la Ciénaga Grande de Santa Marta, Colombia. Boletín de Investigaciones Marinas y Costeras INVEMAR 25 (1): 19-48.

Bernal, G. y Betancur, J. 1996. Sedimentología de Lagunas 128
Pajarales. Boletín de Investigaciones Marinas y Costeras INVEMAR 25 (1): 49-76.

Botero, L. y Mancera, J. E. 1996. Síntesis de los cambios de origen antrópico ocurridos en los últimos 40 años en la Ciénaga Grande de Santa Marta (Colombia). Revista de la Academia Colombiana de Ciencias Exactas, Físicas y Naturales 20: 465-474.

Gaarder, T. y Gran, H. H. 1927. Investigations of the production of plankton in the Oslo Fjord. Rapports et Procès-Verbaux des réunions. Conseil International pour L'Exploration de la Mer 42: 1-48.

Gocke, K., Hernández, C., Giesenhagen, H. y Hoppe, H. 2004. Seasonal variations of bacterial abundance and biomass and their relation to phytoplankton in the hypertrophic tropical lagoon Ciénaga Grande de Santa Marta, Colombia. Journal of Plankton Research 26(12): 1429-1439.

González, S.G. y Berdayes, J.A. 1981. La producción primaria en las lagunas costeras de Tunas de Zaza, Cuba. Revista Investigaciones Marina II (2): 109-139.

Granados-Martínez, C., Rodríguez-Chila, J. y López-Salgado, H. 2011. Producción primaria fitoplanctónica y metabolismo durante una época lluviosa en una laguna costera estuarina tropical. Ciénaga Grande de Santa Marta. Intropica 6: 9-19.

Grasshoff, K., Ehrhardt, M. y Kremling, K. 1983. Methods of Seawater Analysis. Second, Revised and Extended Edition. WILEY-VCH, Weinhem.

Hernández, C. 1986. Producción primaria y dinámica del fitoplancton en la Ciénaga Grande de Santa Marta, Colombia. Tesis de Maestría, Universidad Nacional, Bogotá, Colombia.

Hernández, C. 1988. Producción primaria del fitoplancton en la Ciénaga Grande de Santa Marta, Colombia. Informe técnico, primera parte. INVEMAR - COLCIENCIAS, Santa Marta.

Hernández, C. y Gocke, K. 1990. Productividad primaria en la Ciénaga Grande de Santa Marta. Anales Instituto Investigaciones Marinas de Punta Betín 19-20: 101-119.

IDEAM. 2005. Atlas Climatológico de Colombia. Instituto de Hidrología, Meteorología y Estudios Ambientales, Bogotá.

IGAC. 1993. Características Geográficas Magdalena. Instituto Geográfico Agustín Codazzi, Bogotá.

INVEMAR, 2000. Monitoreo de las condiciones ambientales $y$ los cambios estructurales y funcionales de las comunidades 
vegetales y de recursos pesqueros durante la rehabilitación de la Ciénaga Grande de Santa Marta: Un enfoque de manejo adaptativo. Informe Técnico Final 2001. Ministerio Medio Ambiente-Banco Interamericano Desarrollo INVEMAR, Santa Marta.

Knoppers, B. 1993. Aquatic Primary Production in Coastal Lagoons. En: Kjerfve, B., Editor. Coastal Lagoon Processes. Elservier Oceanography Series, Columbia.

Kucklick, M. 1987. Phytoplankton biomasse und seston grehalt eines mangrove ästuars (Ciénaga Grande de Santa Marta, Kolumbien). Tesis de Doctorado, Universitát Stuttgart Hohenhein Botanisches Institut, Stuttgart, Alemania.

Lecarpentier, C., Umaña, G. y Vega, G. 1975. Estudio hidroclimático de la región del Caribe. IGAC, Bogotá.

Lorenzen, C. J. 1967. Determination of chlorophyll and pheopigments spectronic equations. Limnolgy and Oceanography 12: 343-346.

Margalef, R. 1983. Limnología. Ediciones Omega S.A., Barcelona.

Mogollón, M. 1996. Estructura de la comunidad fitoplanctónica del Complejo de Pajarales y Salamanca Occidental, Costa Caribe Colombiana. Tesis de pregrado, Universidad Jorge Tadeo Lozano, Bogotá, Colombia.

Odum, E. P. y Sarmiento, F. O. 1997. Ecología el puente entre ciencia y sociedad. Editorial Mc-Graw-Hill Interamericana, México D. F.

Parra O. O., M. González, V. Delarosa, P. Rivera y Orellana, M. 1982. Manual taxonómico de fitoplancton de aguas continentales. Con especial referencia al fitoplancton de Chile. 1982. I-Cyanophyceas. Universidad de Concepción, Concepción, Chile.

Pereira I., Reyes, G. y Kramm. V. 2000. Cyanophyceae, Euglenophyceae, Chlorophyceae, Zygnematophyceae y Charophyceae en arrozales de Chile. Gayana Botánica 57: 29-53.

PROCIENAGA. 1994. Estudio de impacto ambiental. Informe final proyecto: Reapertura de canales en el delta exterior derecho del río Magdalena, Santa Marta.

RAMSAR, Ministerio de Ambiente, Vivienda y Desarrollo Territorial, Corporación Autónoma Regional del Magdalena (CORPAMAG) e INVEMAR. 2004. Plan de manejo para el sitio RAMSAR y reserva de la biosfera, sistema delta estuarino del rio Magdalena, Ciénaga Grande de Santa Marta. Talleres de Unión Gráfica Ltda, Bogotá.
Rodríguez-Chila, J. D., Mancera-Pineda, J. E. y LópezSalgado, H. J. 2009. Efectos de la recomunicación del río Magdalena con su antiguo delta: cambios en la producción primaria fitoplanctónica y respiración en el complejo Pajarales, 1989 A 2005. Boletín de Investigaciones Marinas y Costeras INVEMAR 38(2): 119-144.

Rondón, E. 1991. Estimación de la productividad fitoplanctónica en Ciénagas del Complejo de Pajarales, Caribe colombiano. Tesis de Pregrado, Pontificia Universidad Javeriana, Bogotá, Colombia.

Ruiz, D. M. 1999. Valoración cualitativa y cuantitativa del fitoplancton en ciénagas del delta exterior derecho del río Magdalena y su relación con la reapertura del Caño Clarín. Tesis de Pregrado, Universidad Jorge Tadeo Lozano, Bogotá, Colombia.

Ryszowski, L. 1998. Economía de la energía y de la materia en los ecosistemas. En: Dobben, W. H. y Lowe-McConnell, R. H., Editores. Conceptos Unificadores en ecología. Blume Ecología, Barcelona.

Velásquez A. y Rosales C. 2002. Los pasos de El Niño en Colombia 1980 - 2001: Algunas interpretaciones, oportunidades y utilizaciones. Informe Anual Colombia Año \#2, Proyecto ENSO 15 de enero del 2002, LA RED IAI, Universidad del Valle, Cali.

Vidal, L. A. 2010. Manual del fitoplancton hallado en la Ciénaga Grande de Santa Marta y cuerpos de agua aledaños. Bogotá: Fundación Universidad Jorge Tadeo Lozano, Bogotá.

Vidal, L. A. 1995. Estudio de fitoplancton en el sistema lagunar estuarino tropical Ciénaga Grande de Santa Marta. Colombia, durante el año 1987. Tesis Magíster Scientiae Universidad Nacional de Colombia, Bogotá, Colombia.

Vidal, L. A., 1996. Estudio del fitoplancton en la Ciénaga Grande de Santa Marta durante el año de 1995. Resultados cualitativos y variación en el tiempo. Informe Final INVEMAR, Santa Marta.

Vidal, L. A., Guillot, G., Sánchez, G. E. y Criales, M. 2001. Composición del fitoplancton de la Ciénaga Grande de Santa Marta (CGSM) Caribe colombiano, octubre 1999julio 2000. En: IX Congreso Latinoamericano Sobre Ciencias del Mar, Colombia.

Vollenweider, R. A. 1974. A manual on methods for measuring primary production in aquatic environments. IBP Handbook No. 12, Blackwell Scientific, Oxford.

Yacubson, S. 1969. Algas de ambientes acuáticos continentales para Venezuela. Boletín Centro Investigaciones Biológicas (3): 7- 87. 
Yacubson, S. 1972. Catálogo e iconografía de las Cyanophytas de Venezuela. Boletín Centro Investigaciones Biológicas (5): 7- 78.

Yacubson, S. 1974a. Catálogo e iconografía de las Chlorophytas de Venezuela. Boletín Centro Investigaciones Biológicas (11): 7- 143.

Yacubson, S. 1974b. El Fitoplancton de la Laguna de San Javier del Valle (Estado Mérida), Venezuela. Revue Algologique Tome XI Fascicule 1-2: 91-131.

Yacubson, S. 1980. Algas del río Limón y ambientes acuáticos cercanos (Estado Zulia Venezuela). Boletín Centro Investigaciones Biológicas (14): 1- 81.
Yacubson, S. 1984. Algas del río Tucuco y ambientes acuáticos de sus alrededores (Estado Zulia Venezuela). Boletín Centro Investigaciones Biológicas (17): 19- 95.

Yacubson, S. y Bravo, C. R. 1982. Especies de Trachelomonas (Euglenophytas) de algunos cuerpos de agua de los distritos Urdaneta y Perijá (Estado Zulia Venezuela). Boletín Centro Investigaciones Biológicas (15): 17- 47.

Yacubson, S. y Bravo C. R. 1986. Especies de Phacus (Euglenophytas) de diversos cuerpos ambientes acuáticos del Estado de Zulia Venezuela). Boletín Centro Investigaciones Biológicas (17): 47- 77.

Fecha de recepción: 26/07/2017

Fecha de aceptación: 11/09/2017

Publicado en línea: 14/09/2017

Para citar este artículo: Hernández-Jiménez C.A. 2017. Efectos de la entrada de agua del río Magdalena en la producción primaria del fitoplancton en la ciénaga pajaral, Caribe colombiano. Intropica 12(2): 117-130. DOI: http://dx.doi.org/ 10.21676/23897864.2315 